\title{
Investigation of the effect of Staufen 1 overexpression on the HIV-1 virus production
}

\author{
Seong-won Park, Kyung-Lee Yu, Jun-Hyun Bae, Ga-Na Kim, Hae-In Kim E Ji Chang You* \\ National Research Laboratory of Molecular Virology, Department of Pathology, School of Medicine, The Catholic University of Korea, \\ Seoul 63071, Korea
}

\begin{abstract}
In this study, we investigated how Staufen1 influences the HIV-1 production. The overexpression of Staufen 1 increased virus production without any negative affect on the viral infectivity. This increase was not caused by transcriptional activation; but by influencing post-transcriptional steps. Using multiple Gag protein derivatives, we confirmed that the zinc-finger domains of the HIV-1 nucleocapsid (NC) are important for its interaction with Staufen1. We also found that Staufen 1 colocalized in stress granules with the mature form of the HIV-1 NC protein. [BMB Reports 2021; 54(11): 551-556]
\end{abstract}

\section{INTRODUCTION}

Staufen is a double-stranded RNA-binding protein. Mammals have two homologs, Staufen1 and Staufen2, both of which have similar functions (1). Two Staufen 1 isoforms are produced by differential splicing, with molecular weights of 55 and $63 \mathrm{kDa}$.

Staufen1 is known to contribute to multiple cellular processes involving mRNAs. For example, human Staufen1 plays a role in mRNA localization and transportation on microtubules (2). This transport involves the formation of a ribonucleoprotein (RNP) complex generated by Staufen 1 self-association (3). Staufen 1 also contributes to the mRNA decay process in mammalian cells. Staufen1-mediated mRNA decay (SMD) involves interaction with a Staufen 1 binding site (SBS) in the $3^{\prime}$-untranslated regions (UTRs) of target mRNAs, recruiting the ATPdependent RNA helicase UPF1 (4). Studies have shown that SMD contributes to the differentiation of myoblasts (5), as well as to the regulation of adipogenesis, through competition with nonsense-mediated mRNA decay (6). Furthermore, in binding to the $5^{\prime}$ ends of specific mRNAs, Staufen 1 may also enhance

*Corresponding author. Tel: +82-2-2258-7510; Fax: +82-2-2258-7790; E-mail: jiyou@catholic.ac.kr

https://doi.org/10.5483/BMBRep.2021.54.11.072

Received 3 June 2021, Revised 16 June 2021, Accepted 7 July 2021

Keywords: HIV-1, RNA-binding proteins, Staufen1, Virus-host interaction, Virus replication translation (7). Post-transcriptional regulation has been shown to be involved in cell-cycle progression (8). During oxidative stress, Staufen 1 may be recruited to stress granules, leading to their dissolution and the assembly and stabilization of polysomes (9).

Structurally, Staufen 1 has tubulin-binding domains (TBDs) that associate with the cytoskeleton during mRNA transport (10). Staufen 1 has been shown to undergo both homo- and heterodimerization during SMD (11). This process involves the Staufen1 swapping motif (SSM) which resides between the tubulin-binding domain (TBD) and dsRNA-binding domain 5 (RBD5). Recently, Staufen 1 has been shown to contain flexibility linkers that make it possible for the protein to adopt an elongated conformation (12).

A series of studies have investigated the involvement of Staufen 1 in viral replication and interaction. Its association with non-structural protein 1 (NS1) of the influenza A virus was shown to reduce viral replication $(13,14)$. On the other hand, hepatitis $\mathrm{C}$ virus $(\mathrm{HCV})$ protein and RNA levels were found to increase when Staufen1 levels rose. Staufen 1 is also known to promote replication by inhibiting protein kinase $\mathrm{R}$ activity and by its involvement in the transport of HCV RNA $(15,16)$. The human endogenous retrovirus $\mathrm{K}$ (HERV-K) Rec protein interacts with Staufen1, promoting viral RNA transportation and virion production (17). In Ebola virus (EBOV) infection, Staufen1 has been shown to promote RNA synthesis by binding to the $3^{\prime}$ and $5^{\prime}$ extracistronic regions of the EBOV genome, as well as by interacting with viral components such as NP, VP30, and VP35, to form a viral RNP complex (18). The 5'-UTR region of enterovirus 71's RNA has a Staufen 1 binding site, at which interaction facilitates and extends viral RNA stability for translation and replication (19). In summary, Staufen 1 has been shown to interact with many viral proteins and RNAs, contributing to both RNA replication and virus production.

Staufen 1 has been shown to play a role in HIV-1 replication. It is incorporated within HIV-1 particles, with overexpression of the protein enhancing selective HIV-1 genomic RNA encapsidation (20). It has also been reported that both the dsRBD3 and $88 \mathrm{~N}$-terminal amino acids of Staufen 1 are required to enhance Gag multimerization, encapsidation, and virus-like particle (VLP) release (20-23). A Staufen1-HIV-1-dependent ribonucleoprotein (SHRNP) complex has been reported that it is 
distinct from stress granules and processing bodies. This complex prevents degradation of viral RNA and enhances both Gag assembly and RNA packaging (24). It was recently reported that Staufen 1 overexpression caused a reduction in NC proteininduced stress granules, resulting in the rescue of viral mRNA translation by relieving inhibition by elF2alpha phosphorylation, a downstream pathway of PRK that facilitates polysome stabilization (25). In a later study, HIV-1 RNA was mistrafficked to stress granules in Staufen 1 knockout cells, resulting in reduced virus production and impaired infectivity (26).

One study reported that the overexpression of Staufen 1 reduced viral infectivity 6.7-fold (20), while later Staufen 1 knockdown data suggested that it is required for the generation of infectious virus (23). More recently, Staufen $1(-/-)$ cells have been used to show that depletion decreases both virus production and infectivity (26). These results show similarities to the aforementioned case involving Staufen 1 overexpression. This raises questions regarding the exact nature of the relationship between Staufen 1 and virus infectivity.

The present study aims to increase our understanding of the role Staufen 1 in HIV-1 production. We found that Staufen 1 overexpression can increase the production of viruses and their associated proteins, while not generating a negative impact on the viral infectivity. Moreover, the enhancement of virus production by Staufen 1 is not due to transcriptional activation, but rather to post-transcriptional process. We also found that Staufen1 can be co-localized in stress granules with a mature form of the HIV-1 NC protein.

\section{RESULTS AND DISCUSSION}

\section{Generation of A high-expression Staufen 1 construct}

To investigate the role of Staufen1 in HIV-1 replication and production, we first employed a Staufen1 expression vector obtained from Dr. Andrew J Mouland's laboratory (20). However, the expression level of Staufen 1 was deemed to be too low for our analyses. In this plasmid, expression is driven by the respiratory syncytial virus (RSV) promoter. To meet the

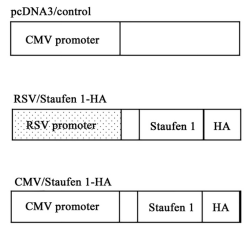

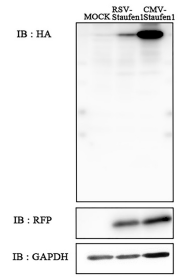

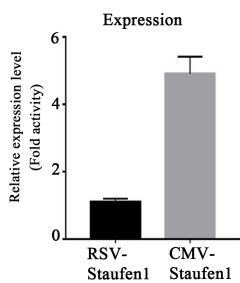

Fig. 1. Construction of a Staufen 1 expression plasmid. (A) Schematics of the control, RSV-Staufen1 and CMV-Staufen1 plasmids. (B) HEK293T cells were transfected RSV-Staufen1 or CMV-Staufen1 with RFP. Cells were harvested after transfection for $24 \mathrm{~h}$. The cell lysate was used for western blotting detection of Staufen1. (C) Measurements of band densities using the Image J program. requirements of our study, we constructed a new expression vector by replacing the RSV promoter with a cytomegalovirus (CMV) promoter, as shown in Fig. 1A. Then, 293T cells were transfected with either the resulting CMV-Staufen1 vector or the original RSV-Staufen1 vector for comparison; we observed that Staufen 1 expression levels in the former had increased greatly 5 -fold or more when compared to the latter (Fig. 1B, C). Thus, this new construct was utilized in subsequent experiments.

\section{Staufen 1 overexpression increases HIV-1 production}

Previous studies have shown that Staufen 1 increases HIV-1 VLP release (22). To examine the effect Staufen 1 overexpression has on protein and virus production levels, 293T cells were co-transfected with CMV-Staufen1 and the proviral vector pNL4.3GFP. A red fluorescent protein (RFP) expression vector was used as an internal control in each transfection assay, with transfection efficiency confirmed by fluorescence microscopy (Fig. 2A). After $24 \mathrm{~h}$, we harvested the cell lysate and supernatant for analysis. Western blotting and enzyme-linked immunosorbent assays (ELISAs) were used to assess protein and virus levels. We found that Gag increased in a dose-dependent manner with Staufen 1 overexpression. Furthermore, we noted
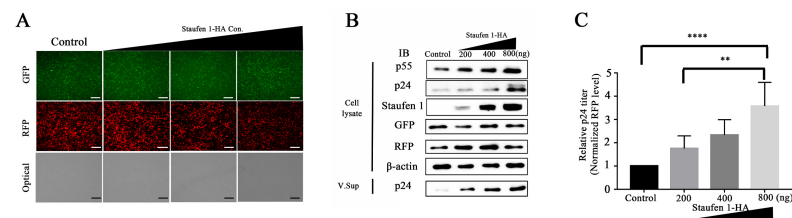

$\mathrm{D}$
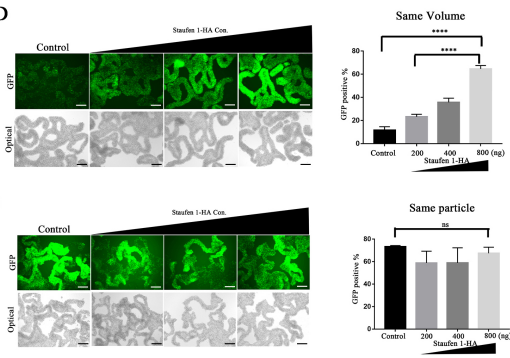

Fig. 2. Increase in virus production due to Staufen 1 overexpression (A-C) HEK293T cells were seeded $2.5 \times 10^{5}$ and cotransfected with proviral DNA pNL4-3 GFP (200 ng) with CMV-Staufen1 followed by different level using the jetPEI. Incubation for $24 \mathrm{~h}$ after transfection. (A) Detection of cell fluorescence using by inverted fluorescence microscopy. Scale bar $2.0 \mathrm{~mm}$. (B) Western blot analysis. (C) Effect of different concentrations of CMV-Staufen 1 on p24 ELISA, normalized to RFP levels. Fold activity compared with control. (D, E) MT4 cells were infected with identical volumes of virus supernatant or same particle of virus for 4 days. Scale bar $2.0 \mathrm{~mm}$ (D) Inverted fluorescence micrograph (left panel) and GFP-positive cells quantified by FACS analysis (right panel). (E) Inverted fluorescence micrograph (left panel) and GFP-positive cells quantified by FACS analysis (right panel). Results are shown as means \pm SD for three independent experiments. Statistical significance was determined using one-way ANOVA $(* * * * P<0.0001, * * \mathrm{P}=0.0029, \mathrm{~ns}=$ not significant). 
that green fluorescent protein (GFP) levels were not significantly changed (Fig. 2B), indicating that only Gag level had increased. ELISA showed that when the Staufen 1 levels increased, the amounts of capsid protein in the viral supernatant increased approximately 3.5-fold over the control (Fig. 2C). As shown in Fig. 2A-C, overexpression of Staufen 1 markedly increased cellular levels of Gag, resulting in an associated increase in virus production.

Previous studies have shown that both overexpression and depletion of Staufen 1 can decrease somehow the infectivity of HIV-1 (20, 23, 26). Therefore, we tested the infectivity of virus produced from 293T cells with and without Staufen 1 overexpression by using culture supernatants (Fig. 2C) to infect MT4 cells. Infection using equal amounts of virus showed clearly that infectivity increased with Staufen 1 overexpression. The intensity of GFP fluorescence in the western blot and ELISA data (Fig. 2D) also supported this observation. These results highlight that Staufen1 increased the production of HIV-1 without an associated any negative effect on the infectivity. To confirm this, we subjected fresh MT4 cells to reinfection with identical amounts of virus from cells with and without Staufen 1 overexpression (Fig. 2E). Subsequent fluorescence microscopy and FACS analysis showed no detectable difference between these two groups, again indicating that Staufen 1 overexpression did not negatively affect the infectivity of HIV-1 generated with Staufen 1 overexpression.

\section{Staufen 1 does not influence HIV-1 LTR promoter activity}

To examine whether the increase in virus production was due to Staufen 1 transcriptional activity, we examined the effect this protein had on the HIV-1 LTR promoter-driven reporter gene. HEK293T cells were transfected with pGL/U3RU5psi/EGFP at increasing concentrations of Staufen1. As shown in Fig. 3, the transfected cells showed similar fluorescence in both the transfection efficiency control and in the presence of increased Staufen 1 levels. Western blotting also demonstrated no significant change (Fig. 3C). These results indicate that even as Staufen1 expression levels increased, the HIV-1 LTR promoter retained normal activity levels.

We also conducted another reporter gene assay using the firefly luciferase (Fluc) gene. We transfected pGLU3RU5psi/Fluc and pCDNA4 TO_EGFP (control) with increasing amounts of the Staufen 1 expression vector. We normalized luciferase activity values to the internal control for GFP intensity, which was found to be similar to the intensity of the enhanced GFP (EGFP) reporter gene (Fig. 3D). Western blotting also showed no increase in Fluc protein level when Staufen1 was overexpressed (Fig. 3E).

Together, these results indicate that Staufen 1 did not affect HIV-1 LTR promoter activity. This confirms that the enhancement of virus production by Staufen 1 has nothing to do with transcription, but instead function(s) post-transcriptionally.
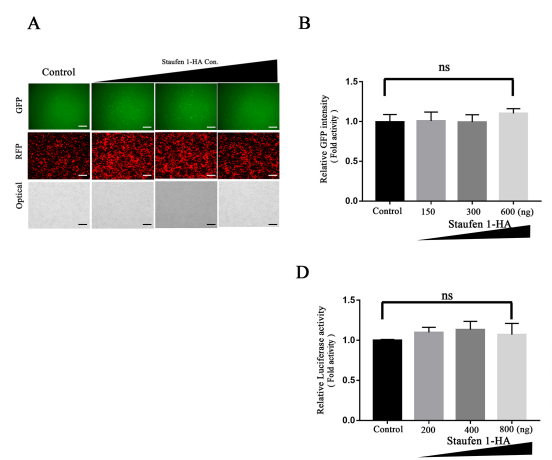

E
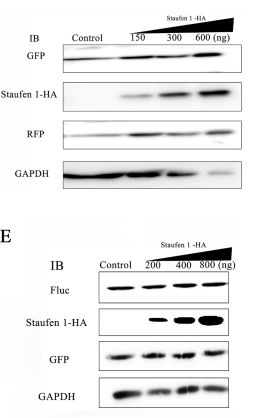

Fig. 3. Lack of Staufen 1 effect on HIV-1 LTR promoter activity. (A-C) HEK293T cells were cotransfected with the reporter gene U3RU5psi-EGFP (200 ng) and CMV-Staufen1 followed by different level using the jetPEI. (A) Inverted fluorescence micrograph. Scale bar $2.0 \mathrm{~mm}$ (B) GFP and RFP intensities of cell lysates measured by fluorometry. (C) Western blot results. (D, E) HEK293T cells were cotransfected with reporter gene DNA, U3RU5psi-Fluc (200 ng), and CMV-Staufen1 at different levels using jetPEI analysis. (D) Fluc intensity of cell lysate measured by fluorometer. (E) Western blot results. Data are shown as means \pm SD for three independent experiments. Statistical significance was tested by one-way ANOVA (ns; not significant).

\section{Staufen1 and HIV-1 NC protein interaction and colocalization}

Previously reported was that Staufen 1 influences the multimerization of Gag, which results in an increase in VLP release (22). It has been reported that NC domain of Gag is required for this interaction. This was shown using a fusion NC protein tagged with a yellow fluorescent protein for BRET and coimmunoprecipitation assays. A mutation in the zinc finger of the $\mathrm{NC}$ domain resulted in decreased Staufen $1 / \mathrm{NC}$ interaction (21-23). Therefore, it appears that the interaction of Staufen 1 and the NC domain of Gag may have a significant effect on HIV-1 replication and production.

To further address further the specific interaction between Staufen 1 and Gag, we employed and investigated a mature form of the NC protein without the presence of any fusion proteins. This allowed us to rule out the involvement of other protein structures. We transfected 293T cells with a Staufen1 expression vector along with multiple expression plasmids including pLP-Gag, pLP-D2 (P2-NC-P1-P6), pLP-NC, and MA $\mathrm{G}_{2 \mathrm{~A}} \mathrm{CA}$ (a myristylation site mutant; MA glycine-to-alanine point mutation). Staufen 1 was found to interact with Gag, D2, and NC when pulled down with anti-NC, but not with $\mathrm{MA}_{\mathrm{G} 2 \mathrm{~A}} \mathrm{CA}$ (Fig. 4A). When pulling down with Staufen1, Gag, D2, and NC co-immunoprecipitated (Fig. 4B). These data demonstrate the interaction between Staufen 1 and NC, as well as with proteins containing an NC domain. Furthermore, we tested the interaction of Staufen 1 with Gag proteins containing a mutation in the zinc finger of the NC domain. HEK293T cells were cotransfected with a Staufen 1 expression plasmid and either pLP-Gag 


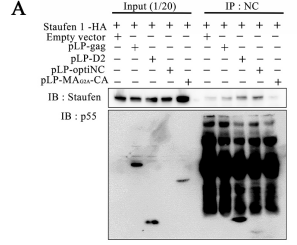

C

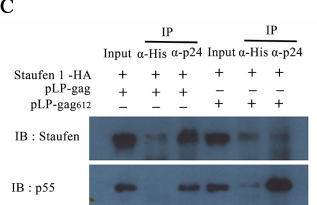

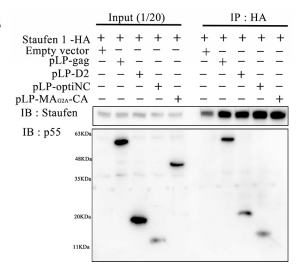

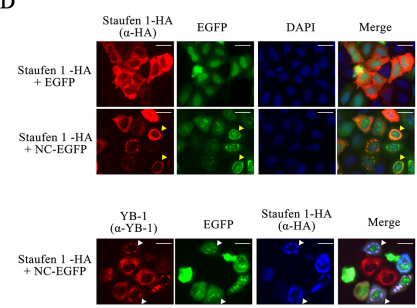

Fig. 4. Interaction and colocalization of Staufen 1 with the NC domain. (A, B) HEK293T cells were transfected pLP1-Gag, and derivatives with CMV-Staufen1, and incubation for $24 \mathrm{~h}$. Coimmunoprecipitation (Co-IP) of Gag, derivatives and CMV-Staufen1. (A) Co-IP performed with rabbit anti-NC antibody. Western blotting using anti-p55 antibody (empty represents the control plasmid). (B) IP performed with mouse anti-HA antibody (CMV-Staufen 1 contains the HA tag). Western blotting using anti-p55 antibody. (C) HEK293T cells were transfected with CMV-staufen-1 and pLP-Gag or pLPGag ${ }^{612}$. Cell lysates subjected to pulldowns with anti-His (negative control) or anti-p24 antibodies. (D) HeLa cells transfected with CMV-Staufen1 and NC-EGFP or EGFP. Scale bar $20 \mu \mathrm{m}$.

or pLP-Gag ${ }^{612}$, which has point mutations (cysteine to serine) in two zinc finger motifs of the NC domain. As shown in Fig. 4C, only wild-type Gag proteins precipitated with Staufen 1. These results confirm affirmatively that the zinc-finger of the NC domain is critical for interaction between Staufen 1 and Gag.

Next, to further investigate the interaction of Staufen 1 and $\mathrm{NC}$ at the subcellular level, we transfected HeLa cells with Staufen 1 and NC-EGFP or EGFP (Fig. 4D). When Staufen1 was expressed alone, it localized to the cytoplasm. However, when Staufen 1 was coexpressed with NC, both proteins colocalized in punctuated forms in the perinuclear area of the cell (Yellow arrows, Fig. 4D). Previous studies highlighted an association between NC and stress-granule components through immunoprecipitation and immunohistochemistry analyses $(25,26)$. We then also examined the Y-box binding protein 1 (YB-1) in transfected HeLa cells, as this is a known stress-granule marker (27, 28). YB-1 was detected along with Staufen 1 and NC in the perinuclear area (white arrows, Fig. 4D), colocalized with stress granules. These results show that there is indeed an interaction between $\mathrm{NC}$ and Staufen 1 that requires the $\mathrm{NC}$ zinc-finger motif. Moreover, this specific interaction could influence Staufen 1 localization, resulting in NC and colocalization in stress granules.

\section{MATERIALS AND METHODS}

\section{Cell culture and transfection}

HeLa and HEK293T cells were obtained from the American Type Culture Collection (ATCC). Cells were cultured in Dulbecco's modified Eagle's medium (DMEM) containing 10\% fetal bovine serum (TCB, USA), penicillin, and streptomycin (GIBCO, Carlsbad, CA, USA). MT4 cells were cultured in Roswell Park Memorial Institute (RPMI) 1640 medium containing an identical serum and antibiotic composition. The cells were incubated in $5 \% \mathrm{CO}_{2}$ at $37^{\circ} \mathrm{C}$. HEK293T and HeLa cells were transfected with plasmid DNA using pMAX (Aptabio Co., South Korea), following the manufacturer's protocol.

\section{Plasmids}

The RSV-Staufen1-HA plasmid DNA was kindly provided by Dr. Mouland. We cloned the CMV-Staufen1-HA plasmid using the DNA restriction enzymes Bg/II and HindlII to isolate the RSV-Staufen1-HA promoter fragment. The CMV promoter was inserted from the pCDNA3-control plasmid using T4 DNA ligase (Elpis Biotech Corp., South Korea). Lastly, pDS-RedExpression-N1 was used as the RFP expression vector.

\section{Reporter gene assays}

HEK293T cells were transfected with U3RU5psi-EGFP and an RFP expression vector. They were then incubated for $48 \mathrm{~h}$. Cells were harvested and lysed with radioimmunoprecipitation assay (RIPA) buffer. EGFP and RFP levels were measured using a fluorometer (Tecan Corp., Switzerland). EGFP levels were normalized to RFP levels. For the luciferase assay, HEK293T cells were transfected with U3RU5psi-Fluc and pCDNA4-TOEGFP, then lysed using passive lysis buffer (Promega Corp., USA) $24 \mathrm{~h}$ after transfection. Luciferase activity was normalized to GFP levels.

\section{Westem blotting}

Cells were lysed using RIPA or passive lysis buffers (Promega Corp., USA). Lysates were boiled in SDS sample buffer for approximately $5 \mathrm{~min}$. Each sample was separated on 12 or $13.5 \%$ SDS-PAGE gels. The gels were then transferred to a PVDF membrane (Merck Millipore Corp. Billerica, MA, USA) and blocked with Tris-buffered saline containing $0.1 \%$ Tween 20 (TBST) and 5\% skim milk for approximately $1 \mathrm{~h}$ at room temperature. The membrane was incubated with the appropriate primary antibody overnight at $4^{\circ} \mathrm{C}$, for $2 \mathrm{~h}$ at room temperature, followed by further incubation with the appropriate secondary antibodies. The protein band signal was developed using the enhanced chemiluminescence system LAS4000.

\section{Immunofluorescence (IF) analysis}

We seeded HeLa cells on coverslips at $4 \times 10^{4} /$ well in 24-well tissue culture plates. These cells were transfected with CMV/ Staufen1-HA, EGFP, or NC-EGFP plasmid DNA using jetPEl. After $24 \mathrm{~h}$, the cells were washed with PBS, fixed with $4 \%$ 
paraformaldehyde, permeabilized for $20 \mathrm{~min}$, blocked for 30 min in $0.1 \%$ Triton $\mathrm{X}-100,2 \%$ bovine serum albumin, and $5 \%$ normal horse serum, and incubated with the primary antibody for $2 \mathrm{~h}$ at room temperature. Next, cells were washed three times and incubated with fluorescent-conjugated secondary antibody for $1 \mathrm{~h}$ while under light protection. After a second round of washing, cells were counterstained with DAPI (1 $\mu \mathrm{g} / \mathrm{ml}$ in PBS-T) for the detection of nuclei. Finally, coverslips were mounted on glass slides using mounting medium and sealed with nail polish.

\section{HIV-1 p24 ELISA}

Cells were transfected with the indicated plasmid DNAs and pNL4-3 EGFP, then supernatants were harvested after $24 \mathrm{~h}$. The amount of p24 in each supernatant was measured using a HIV-1 p24 ELISA kit (Xpressbio, Frederick MD, USA) according to the manufacturer's protocol. This value was normalized to RFP levels.

\section{Coimmunoprecipitation}

For the Staufen 1 and NC protein interaction study, HEK293T cells were transfected with the indicated plasmid DNAs and harvested after a $24 \mathrm{~h}$ incubation. Cells were lysed using NP-40 lysis buffer $(50 \mathrm{mM}$ Tris, $150 \mathrm{mM} \mathrm{NaCl}, 0.5 \%$ Nonidet P-40, $1 \mathrm{mM}$ EGTA). The same volumes of samples were incubated with a specific antibody for $1 \mathrm{~h}$ at $4^{\circ} \mathrm{C}$. Then, the sample and antibody mixtures were incubated with $\mathrm{A} / \mathrm{G}$ agarose beads (Santa Cruz) for $1 \mathrm{~h}$ (or overnight) at $4^{\circ} \mathrm{C}$. The beads were washed three times with PBS and boiled in SDS sample buffer. Protein detection was performed using western blotting.

\section{Antibodies}

Anti-HA monoclonal antibody (Sigma Aldrich Co.), Anti-p24 monoclonal antibody (Santacruz), Anti-GFP polyclonal antibody (Santacruz), Anti-RFP polyclonal antibody (TaKaRa bio Inc.), AntiFirefly antibody monoclonal (Calbiochem), Anti-NC antibody polyclonal (custom antibody), Anti-His monoclonal antibody (Santacruz).

\section{ACKNOWLEDGEMENTS}

This work was supported by a research foundation of Korea (NRF) Grant funded by the Korean Government (2020R1F1A 1075725 and 2017R1A5A1015366).

\section{CONFLICTS OF INTEREST}

The authors have no conflicting interests.

\section{REFERENCES}

1. Furic L, Maher-Laporte $M$ and DesGroseillers L (2008) A genome-wide approach identifies distinct but overlapping subsets of cellular mRNAs associated with Staufen1- and
Staufen2-containing ribonucleoprotein complexes. RNA 14, 324-335

2. Kohrmann M, Luo M, Kaether C, DesGroseillers L, Dotti CG and Kiebler MA (1999) Microtubule-dependent recruitment of Staufen-green fluorescent protein into large RNAcontaining granules and subsequent dendritic transport in living hippocampal neurons. Mol Biol Cell 10, 2945-2953

3. Martel C, Dugre-Brisson S, Boulay K et al (2010) Multimerization of Staufen1 in live cells. RNA 16, 585-597

4. Kim YK, Furic L, Desgroseillers L and Maquat LE (2005) Mammalian Staufen1 recruits Upf1 to specific mRNA 3'UTRs so as to elicit mRNA decay. Cell 120,195-208

5. Ravel-Chapuis A, Crawford TE, Blais-Crepeau ML, Belanger G, Richer CT and Jasmin BJ (2014) The RNA-binding protein Staufen 1 impairs myogenic differentiation via a c-mycdependent mechanism. Mol Biol Cell 25, 3765-3778

6. Cho H, Kim KM, Han S et al (2012) Staufen1-mediated mRNA decay functions in adipogenesis. Mol Cell 46, 495506

7. Dugre-Brisson S, Elvira G, Boulay K, Chatel-Chaix L, Mouland AJ and DesGroseillers L (2005) Interaction of Staufen 1 with the $5^{\prime}$ end of mRNA facilitates translation of these RNAs. Nucleic Acids Res 33, 4797-4812

8. Boulay K, Ghram M, Viranaicken W et al (2014) Cell cycle-dependent regulation of the RNA-binding protein Staufen1. Nucleic Acids Res 42, 7867-7883

9. Thomas MG, Martinez Tosar LJ, Desbats MA, Leishman CC and Boccaccio GL (2009) Mammalian Staufen 1 is recruited to stress granules and impairs their assembly. J Cell Sci 122, 563-573

10. Wickham L, Duchaine T, Luo M, Nabi IR and DesGroseillers L (1999) Mammalian staufen is a double-stranded-RNAand tubulin-binding protein which localizes to the rough endoplasmic reticulum. Mol Cell Biol 19, 2220-2230

11. Gleghorn ML, Gong C, Kielkopf CL and Maquat LE (2013) Staufen1 dimerizes through a conserved motif and a degenerate dsRNA-binding domain to promote mRNA decay. Nat Struct Mol Biol 20, 515-524

12. Visentin S, Cannone G, Doutch J et al (2020) A multipronged approach to understanding the form and function of hStaufen protein. RNA 26, 265-277

13. de Lucas S, Peredo J, Marion RM, Sanchez C and Ortin J (2010) Human Staufen1 protein interacts with influenza virus ribonucleoproteins and is required for efficient virus multiplication. J Virol 84, 7603-7612

14. Lee JH, Oh JY, Pascua PN, Kim EG, Choi YK and Kim HK (2011) Impairment of the Staufen1-NS1 interaction reduces influenza viral replication. Biochem Biophys Res Commun 414, 153-158

15. Dixit U, Pandey AK, Mishra P, Sengupta A and Pandey VN (2016) Staufen 1 promotes HCV replication by inhibiting protein kinase $\mathrm{R}$ and transporting viral RNA to the site of translation and replication in the cells. Nucleic Acids Res 44, 5271-5287

16. Blackham SL and McGarvey MJ (2013) A host cell RNAinding protein, Staufen1, has a role in hepatitis $C$ virus replication before virus assembly. J Gen Virol 94, 24292436

17. Hanke K, Hohn O, Liedgens L et al (2013) Staufen-1 interacts with the human endogenous retrovirus family 
HERV-K(HML-2) rec and gag proteins and increases virion production. J Virol 87, 11019-11030

18. Fang J, Pietzsch C, Ramanathan P et al (2018) Staufen1 interacts with multiple components of the Ebola virus ribonucleoprotein and enhances viral RNA synthesis. mBio 9, e01771-18

19. Chen YM, Ou BT, Chen CY, Chan HH, Chen CJ and Wang RY (2019) Staufen1 protein participates positively in the viral RNA replication of Enterovirus 71. Viruses 11, 142

20. Mouland AJ, Mercier J, Luo M, Bernier L, DesGroseillers L and Cohen EA (2000) The double-stranded RNA-binding protein Staufen is incorporated in human immunodeficiency virus type 1: evidence for a role in genomic RNA encapsidation. J Virol 74, 5441-5451

21. Chatel-Chaix L, Boulay K, Mouland AJ and Desgroseillers $L$ (2008) The host protein Staufen 1 interacts with the Pr55Gag zinc fingers and regulates HIV-1 assembly via its $\mathrm{N}$-terminus. Retrovirology 5, 41

22. Chatel-Chaix L, Abrahamyan L, Frechina C, Mouland AJ and DesGroseillers L (2007) The host protein Staufen1 participates in human immunodeficiency virus type 1 assembly in live cells by influencing pr55Gag multimerization. J Virol 81, 6216-6230
23. Chatel-Chaix L, Clement JF, Martel C et al (2004) Identification of Staufen in the human immunodeficiency virus type $1 \mathrm{Gag}$ ribonucleoprotein complex and a role in generating infectious viral particles. Mol Cell Biol 24, 2637-2648

24. Abrahamyan LG, Chatel-Chaix L, Ajamian L et al (2010) Novel Staufen 1 ribonucleoproteins prevent formation of stress granules but favour encapsidation of HIV-1 genomic RNA. J Cell Sci 123, 369-383

25. Rao S, Cinti A, Temzi A, Amorim R, You JC and Mouland AJ (2018) HIV-1 NC-induced stress granule assembly and translation arrest are inhibited by the dsRNA binding protein Staufen1. RNA 24, 219-236

26. Rao S, Hassine S, Monette A, Amorim R, DesGroseillers L and Mouland AJ (2019) HIV-1 requires Staufen1 to dissociate stress granules and to produce infectious viral particles. RNA 25, 727-736

27. Chernov KG, Barbet A, Hamon L, Ovchinnikov LP, Curmi PA and Pastre D (2009) Role of microtubules in stress granule assembly: microtubule dynamical instability favors the formation of micrometric stress granules in cells. J Biol Chem 284, 36569-36580

28. Somasekharan SP, El-Naggar A, Leprivier G et al (2015) YB-1 regulates stress granule formation and tumor progression by translationally activating G3BP1. J Cell Biol 208, 913-929 